\title{
Galerkin method applied to telegraph integro-differential equation with a weighted integral condition
}

\author{
A Guezane-Lakoud', N Bendjazia 1* and R Khaldi²
}

\section{"Correspondence:}

bendjazianassima@yahoo.fr

${ }^{1}$ Faculty of Sciences, Laboratory of

Advanced Materials, Badji

Mokhtar-Annaba University, P.O. Box

12, Annaba, 23000, Algeria

Full list of author information is

available at the end of the article

\begin{abstract}
In this work, we study a telegraph integro-differential equation with a weighted integral condition. By means of the Galerkin method, we establish the existence and uniqueness of a generalized solution.
\end{abstract}

MSC: 35L05; 35L20; 35L99

Keywords: integro-differential equation; integral conditions; approximate solution; Galerkin method

\section{Introduction}

In this work, we consider the following hyperbolic integro-differential equation with integral conditions:

$$
l u=\frac{\partial^{2} u}{\partial t^{2}}-a^{2}(x, t) \frac{\partial^{2} u}{\partial x^{2}}+c(x, t) u=f(x, t)+\int_{0}^{t} \alpha(t-s) K(s, u(x, s)) d s
$$

for all $(x, t) \in Q=(0,1) \times(0, T)$, subject to the initial conditions

$$
u(x, 0)=\varphi(x), \quad u_{t}(x, 0)=\psi(x)
$$

and the weighted integral conditions

$$
\begin{aligned}
& \int_{0}^{1} u(x, t) d x=0 \\
& \int_{0}^{1} h(x) u_{t}(x, t) d x=0
\end{aligned}
$$

where $f, \varphi, \psi, h, a, c, \alpha$ and $K$ are given functions.

Various problems arising in heat conduction [1-5], chemical engineering [6], thermoelasticity [7], and plasma physics [8] can be modeled by the nonlocal problems. Boundary value problems with integral conditions constitute a very interesting and important class of problems. These nonlocal conditions arise mostly when the data on the boundary cannot be measured directly. Recall that the presence of an integral term in boundary conditions can complicate the application of classical methods of functional analysis in the

\section{照 Springer}

(c) 2013 Guezane-Lakoud et al.; licensee Springer. This is an Open Access article distributed under the terms of the Creative Commons Attribution License (http://creativecommons.org/licenses/by/2.0), which permits unrestricted use, distribution, and reproduction in any medium, provided the original work is properly cited. 
theoretical study of nonlocal problems, therefore, several methods have been proposed for overcoming the difficulties arising from nonlocal conditions; see Beilin [1], Cannon $e t$ al. [2, 8], and Dehghan et al. [3, 4, 9].

Numerical solutions are introduced to obtain approximations for the solution of partial differential equations when the analytical solutions are difficult or impossible to obtain due to complicated geometry or boundary conditions. In the area of numerical analysis, the Galerkin method is a class of methods for converting a continuous operator problem to a discrete problem. In principle, it is the equivalent of applying the method of a variation of parameters to a function space, by converting the equation to a weak formulation, hence in this approach we choose a system of linearly independent functions such that they satisfy the given homogeneous boundary condition, and they are dense in a function space containing the exact solution of the above boundary value problem.

The advantage of this approach is not only to establish the existence and uniqueness of the solution, but it is also a very effective method in the study of the approximate solution and its convergence.

In this paper, we study the hyperbolic integro-differential equation (1.1) with a Volterra operator of the form $\int_{0}^{t} \alpha(t-s) K(s, u(x, s)) d s$ in the second member, which appears in the modelling of the quasi-static flexure of a thermo-elastic rod and has been studied in [9, 10] under different boundary conditions, by means of the Rothe method. Let us mention that different methods are used to solve similar integro-differential equations, for example, in $[11,12]$ the authors have established the existence and uniqueness of the solution using Rothe's method of an integro-differential equation. In [10,13], the authors have used Rothe's method and the techniques of [7] to prove the existence, uniqueness and continuous dependence of a strong solution to a quasi-linear integro-differential equation. In [6], the local existence and uniqueness of a classical solution of an abstract second-order integro-differential equation in a Banach space have been investigated by using the theory of an analytic semi-groups and contraction mapping theorem. In $[14,15]$ the authors investigated a telegraph equation with non-local integral conditions by means of the Galerkin method.

This paper is organized as follows: In the next section, we define the generalized solution and the functional spaces. In Section 3, we prove that the generalized solution if it exists is unique. The existence of the generalized solution by using the Galerkin method is established in the fourth section, and for this, we construct an approximation solution of the problem (1.1)-(1.4). We prove that we can extract a subsequence, which converges to the desired generalized solution. An application is included to illustrate that corresponding assumptions are satisfied.

\section{Notation and definition}

Let $L^{2}(0,1)$ be the usual space of Lebesgue square integrable real functions on $(0,1)$ whose inner product and norm will be denoted respectively by $(\cdot, \cdot)$ and $\|\cdot\|$. Denote by $H(Q)$ the Sobolev space consisting of all functions $u \in L^{2}(Q)$ having weak derivatives in $L^{2}(Q)$, with the norm

$$
\|u\|_{H(Q)}^{2}=\int_{0}^{T} \int_{0}^{1}\left[\left(\int_{x}^{1} u(\xi, t) d \xi\right)^{2}+(u(x, t))^{2}+\left(\int_{x}^{1} u_{t}(\xi, t) d \xi\right)^{2}\right] d x d t .
$$


Let us define the generalized solution of the problem (1.1)-(1.4). Suppose that $u$ is a solution of this problem, multiply both sides of equation (1.1) by $\int_{x}^{1}(h(\xi)-h(x)) v_{t}(\xi, t) d \xi$, where $v \in H_{T}(Q)=\left\{v(x, t) \in H(Q), v_{t}(x, T)=0\right\}$, integrate by parts the resultant equation over the domain $Q$, use the conditions (1.2), (1.3), (1.4) and the fact that $v_{t}(x, T)=0$, we obtain

$$
\int_{Q} l u\left(\int_{x}^{1}(h(\xi)-h(x)) v_{t}(\xi, t) d \xi\right) d x d t=I_{1}-I_{2}+I_{3}
$$

where

$$
\begin{aligned}
I_{1}= & \int_{Q} u_{t t}(x, t)\left(\int_{x}^{1}(h(\xi)-h(x)) v_{t}(\xi, t) d \xi\right) d x d t \\
= & -\int_{Q} h^{\prime}(x)\left(\int_{x}^{1} u_{t} d \xi\right)\left(\int_{x}^{1} v_{t t}(\xi, t) d \xi\right) d x d t \\
I_{2}= & \int_{Q} a^{2}(x, t) u_{x x}(x, t)\left(\int_{x}^{1}(h(\xi)-h(x)) v_{t}(\xi, t) d \xi\right) d x d t \\
= & 2 \int_{Q}\left(a a_{x}\right)_{x} u\left(\int_{x}^{1}(h(\xi)-h(x)) v_{t}(\xi, t) d \xi\right) d x d t \\
& -\int_{Q}\left(4 a a_{x} h^{\prime}(x)+a^{2} h^{(2)}(x)\right) u\left(\int_{x}^{1} v_{t}(\xi, t) d \xi\right) d x d t+\int_{Q} a^{2} h^{\prime}(x) u v_{t} d x d t
\end{aligned}
$$

and

$$
\begin{aligned}
I_{3}= & \int_{Q} c(x, t) u(x, t)\left(\int_{x}^{1}(h(\xi)-h(x)) v_{t}(\xi, t) d \xi\right) d x d t \\
= & -\int_{Q} c h^{\prime}(x)\left(\int_{x}^{1} u(\xi, t) d \xi\right)\left(\int_{x}^{1} v_{t}(\xi, t) d \xi\right) d x d t \\
& +\int_{Q} c_{x}\left(\int_{x}^{1} u(\xi, t) d \xi\right)\left(\int_{x}^{1}(h(\xi)-h(x)) v_{t}(\xi, t) d \xi\right) d x d t .
\end{aligned}
$$

Calculating $I_{1}-I_{2}+I_{3}$, we deduce

$$
\begin{aligned}
\int_{Q}[- & h^{\prime}(x) \int_{x}^{1} u_{t} d \xi \int_{x}^{1} v_{t t}(\xi, t) d \xi-\left(4 a a_{x} h^{\prime}(x)+a^{2} h^{(2)}(x)\right) u \int_{x}^{1} v_{t}(\xi, t) d \xi \\
& -a^{2} h^{\prime}(x) u v_{t}-c h^{\prime}(x) \int_{x}^{1} u(\xi, t) d \xi \int_{x}^{1} v_{t}(\xi, t) d \xi \\
& +2\left(a a_{x}\right)_{x} u \int_{x}^{1}(h(\xi)-h(x)) v_{t}(\xi, t) d \xi \\
& \left.+c_{x}\left(\int_{x}^{1} u(\xi, t) d \xi\right)\left(\int_{x}^{1}(h(\xi)-h(x)) v_{t}(\xi, t) d \xi\right)\right] d x d t \\
= & \int_{Q} f(x, t)\left(\int_{x}^{1}(h(\xi)-h(x)) v_{t}(\xi, t) d \xi\right) d x d t \\
& +\int_{Q}\left(\int_{0}^{t} \alpha(t-s) K(s, u(x, s)) d s\right)\left(\int_{x}^{1}(h(\xi)-h(x)) v_{t}(\xi, t) d \xi\right) d x d t .
\end{aligned}
$$


Definition 1 By a generalized solution of problem (1.1)-(1.4), we mean a function $u \in$ $H(Q)$ satisfying for all $v \in H_{T}(Q)$ the identity (2.1).

\section{Uniqueness of generalized solution}

For solving the problem, we make the following hypotheses:

(H1) The functions $a$ and $c$ are nonnegative and satisfy on $Q$

$$
\begin{aligned}
& 0<a_{0} \leq a(x, t) \leq A_{0}, \quad\left|a_{t}, a_{x}, a_{x t}, a_{x x}, a_{x x x}\right| \leq A_{1}, \\
& 0<c_{0} \leq c(x, t) \leq C_{0}, \quad\left|c_{x}, c_{t}\right| \leq C_{1} .
\end{aligned}
$$

The function $\alpha$ is continuous and denote $l=\max _{0 \leq t \leq T}|\alpha(t)|$.

(H2) The function $h \in C^{2}([0,1], \mathbb{R}), h^{\prime}$ is nonnegative and satisfy for all $x \in(0,1)$

$$
\left(|h(x)|, h^{\prime}(x),\left|h^{(2)}(x)\right|\right) \leq k_{1}, \quad 0<k_{0} \leq h^{\prime}(x) .
$$

(H3) The operator $K(t, u(x, t))$ is linear with respect to $u$ and continuous according to the both variables $t$ and $u$ and satisfies for all $u \in H(Q)$ and $(x, t) \in Q$

$$
|K(t, u(x, t))| \leq|u(x, t)|
$$

Now we shall show that the generalized solution of problem (1.1)-(1.4) if it exists is unique.

Theorem 2 Assume that $f \in L_{2}(Q), \varphi, \psi \in L_{2}(0,1)$ and hypotheses $(\mathrm{H} 1)-(\mathrm{H} 3)$ hold, then the generalized solution of problem (1.1)-(1.4) if it exists is unique.

Proof Suppose that there exists two different generalized solutions $u_{1}$ and $u_{2}$ of the problem (1.1)-(1.4), then $u=u_{1}-u_{2}$ is a generalized solution of the problem (1.1)-(1.4) with $\varphi=\psi=0$ and second member $F=\int_{0}^{t} \alpha(t-s)\left(K\left(s, u_{1}(x, s)\right)-K\left(s, u_{2}(x, s)\right)\right) d s$. We shall prove that $u=0$ in $Q$. Let $v \in H_{T}(Q)$ and denote for $0 \leq \tau \leq T$.

$$
Q^{\tau}=\{(x, t) \in Q, 0<x<1,0<t \leq \tau\} \text {. Consider the function } v \text { such that }
$$

$$
v(x, t)= \begin{cases}u(x, t), & 0 \leq t \leq \tau \\ 0, & \tau \leq t \leq T .\end{cases}
$$

Substituting $v$ into identity (2.1), it follows

$$
\begin{aligned}
& \int_{Q}\left[-h^{\prime}(x) \int_{x}^{1} u_{t} d \xi \int_{x}^{1} u_{t t}(\xi, t) d \xi-\left(4 a a_{x} h^{\prime}(x)+a^{2} h^{(2)}(x)\right) u \int_{x}^{1} u_{t}(\xi, t) d \xi\right. \\
& \quad-a^{2} h^{\prime}(x) u u_{t}-c h^{\prime}(x) \int_{x}^{1} u(\xi, t) d \xi \int_{x}^{1} u_{t}(\xi, t) d \xi \\
& \quad+2\left(a a_{x}\right)_{x} u \int_{x}^{1}(h(\xi)-h(x)) u_{t}(\xi, t) d \xi \\
& \left.\quad+c_{x}\left(\int_{x}^{1} u(\xi, t) d \xi\right)\left(\int_{x}^{1}(h(\xi)-h(x)) u_{t}(\xi, t) d \xi\right)\right] d x d t
\end{aligned}
$$




$$
\begin{aligned}
& =\int_{Q}\left(\int_{0}^{t} \alpha(t-s)\left(K\left(s, u_{1}(x, s)\right)-K\left(s, u_{2}(x, s)\right)\right) d s\right) \\
& \quad \times\left(\int_{x}^{1}(h(\xi)-h(x)) u_{t}(\xi, t) d \xi\right) d x d t .
\end{aligned}
$$

Integrating by parts it yields

$$
\begin{aligned}
\int_{0}^{1} h^{\prime}(x) & {\left[\left(\int_{x}^{1} u_{t}(\xi, \tau) d \xi\right)^{2}+(a(x, \tau))^{2}(u(x, \tau))^{2}+c(x, \tau)\left(\int_{x}^{1} u(\xi, \tau) d \xi\right)^{2}\right] d x } \\
= & \int_{Q^{\tau}}\left[h^{\prime} c_{t}\left(\int_{x}^{1} u(\xi, t) d \xi\right)^{2}+2 h^{\prime} a a_{t}(u(x, t))^{2}\right. \\
& -\left(8 a a_{x} h^{\prime}(x)+2 a^{2} h^{(2)}(x)\right) u \int_{x}^{1} u_{t}(\xi, t) d \xi \\
& -4\left(a a_{x}\right)_{x} u \int_{x}^{1}(h(\xi)-h(x)) u_{t}(\xi, t) d \xi \\
& \left.+2 c_{x}\left(\int_{x}^{1} u(\xi, t) d \xi\right)\left(\int_{x}^{1}(h(\xi)-h(x)) u_{t}(\xi, t) d \xi\right)\right] d x d t \\
& +2 \int_{Q^{\tau}}\left(\int_{0}^{t} \alpha(t-s)\left(K\left(s, u_{1}(x, s)\right)-K\left(s, u_{2}(x, s)\right)\right) d s\right) \\
& \times\left(\int_{x}^{1}(h(x)-h(\xi)) u_{t}(\xi, t) d \xi\right) d x d t .
\end{aligned}
$$

Applying Cauchy inequality, $\epsilon$-inequality and the hypotheses on the operator $K$ to the last term in the right-hand side of (3.2), we get

$$
\begin{aligned}
& 2 \mid \int_{Q^{\tau}}\left(\int_{0}^{t} \alpha(t-s)\left(K\left(s, u_{1}(x, s)\right)-K\left(s, u_{2}(x, s)\right)\right) d s\right) \\
& \times\left(\int_{x}^{1}(h(\xi)-h(x)) u_{t}(\xi, t) d \xi\right) d x d t \mid \\
& \leq 2\left(\int_{Q^{\tau}}\left|\int_{0}^{t} \alpha(t-s)\left(K\left(s, u_{1}(x, s)\right)-K\left(s, u_{2}(x, s)\right)\right) d s\right|^{2} d x d t\right)^{\frac{1}{2}} \\
& \times\left(\int_{Q^{\tau}}\left|\int_{x}^{1}(h(\xi)-h(x)) u_{t}(\xi, t) d \xi\right|^{2} d x d t\right)^{\frac{1}{2}} \\
& \leq(\max |\alpha(t)|)^{2} \int_{Q^{\tau}}\left|\int_{0}^{t}\left(K\left(s, u_{1}(x, s)\right)-K\left(s, u_{2}(x, s)\right)\right) d s\right|^{2} d x d t \\
& \quad+4 k_{1}^{2} \int_{Q^{\tau}}\left(\int_{x}^{1} u_{t}(\xi, t) d \xi\right)^{2} d x d t \\
& \leq l^{2} \tau \int_{Q^{\tau}}\left|K\left(s, u_{1}(x, s)\right)-K\left(s, u_{2}(x, s)\right)\right|^{2} d s d x \\
& \quad+4 k_{1}^{2} \int_{Q^{\tau}}\left(\int_{x}^{1} u_{t}(\xi, t) d \xi\right)^{2} d x d t \\
& \leq l^{2} \tau \mid K\left(t, u_{1}\right)-K\left(t, u_{2}\right) \|_{L^{2}\left(Q^{\tau}\right)}^{2}+4 k_{1}^{2} \int_{Q^{\tau}}\left(\int_{x}^{1} u_{t}(\xi, t) d \xi\right)^{2} d x d t
\end{aligned}
$$




$$
\begin{aligned}
& \leq l^{2} \tau\left\|u_{1}-u_{2}\right\|_{L^{2}\left(Q^{\tau}\right)}^{2}+4 k_{1}^{2} \int_{Q^{\tau}}\left(\int_{x}^{1} u_{t}(\xi, t) d \xi\right)^{2} d x d t \\
& \leq l^{2} \tau\|u\|_{L^{2}\left(Q^{\tau}\right)}^{2}+4 k_{1}^{2} \int_{Q^{\tau}}\left(\int_{x}^{1} u_{t}(\xi, t) d \xi\right)^{2} d x d t
\end{aligned}
$$

Applying similar inequalities with $\varepsilon=1$, for the second, the third and the fourth terms in the right-hand side of (3.1) then using conditions (H1)-(H3), we obtain

$$
\begin{aligned}
\int_{0}^{1} k_{0} & {\left[\left(\int_{x}^{1} u_{t}(\xi, \tau) d \xi\right)^{2}+a_{0}^{2}|u(x, \tau)|^{2}+c_{0}\left(\int_{x}^{1} u(\xi, \tau) d \xi\right)^{2}\right] d x } \\
\leq & \int_{Q^{\tau}}\left[\left(k_{1}\left(10 A_{0} A_{1}+A_{0}^{2}+4 A_{1}^{2}\right)+l^{2} T\right)|u(x, t)|^{2}\right. \\
& +\left(k_{1}\left(3 C_{1}+4 A_{0} A_{1}+4 A_{1}^{2}\right)+4 k_{1}^{2}\right)\left(\int_{x}^{1} u_{t}(\xi, t) d \xi\right)^{2} \\
& \left.+3 k_{1} C_{1}\left(\int_{x}^{1} u(\xi, t) d \xi\right)^{2}\right] d x d t,
\end{aligned}
$$

denote

$$
\begin{aligned}
& M=k_{1} \max \left(6 A_{0} A_{1}+A_{0}^{2}+\frac{l^{2} T}{k_{1}}, 3 C_{1}+4 k_{1}\right), \\
& m=k_{0} \min \left(1, a_{0}^{2}, c_{0}\right), \quad L=\frac{M}{m}
\end{aligned}
$$

then (3.3) becomes

$$
\begin{aligned}
& \int_{0}^{1}\left[\left(\int_{x}^{1} u_{t}(\xi, \tau) d \xi\right)^{2} d x+|u(x, \tau)|^{2}+\left(\int_{x}^{1} u(\xi, \tau) d \xi\right)^{2}\right] d x \\
& \quad \leq L \int_{Q^{\tau}}\left[\left(\int_{x}^{1} u_{t}(\xi, t) d \xi\right)^{2}+|u(x, t)|^{2}+\left(\int_{x}^{1} u(x, t) d \xi\right)^{2}\right] d x d t
\end{aligned}
$$

Gronwall inequality implies

$$
\int_{0}^{1}\left[\left(\int_{x}^{1} u_{t}(\xi, \tau) d \xi\right)^{2} d x+|u(x, \tau)|^{2}+\left(\int_{x}^{1} u(\xi, \tau) d \xi\right)^{2}\right] d x \leq 0
$$

hence $u(x, \tau)=0$, for all $x \in(0,1)$ and $\tau \in(0, T)$, then $u=0$ in $Q$. Thus, the uniqueness is proved.

\section{Existence of generalized solution}

In order to prove the existence of the generalized solution we apply Galerkin method.

Theorem 3 Assume that the assumptions of Theorem 2 hold, then the problem (1.1)-(1.4) has a unique solution $u \in H(Q)$.

Proof Let $\left\{w_{k}(x)\right\}$ be a fundamental system in $H(0,1)$, such that

$$
\left(w_{k}, w_{i}\right)=\delta_{k, i}= \begin{cases}1, & k=i \\ 0, & k \neq i\end{cases}
$$


We have to find for each $n \in \mathbb{N}^{*}$, the approximate solution of the problem (1.1)-(1.4) which has the following form:

$$
u^{(n)}=\sum_{k=1}^{n} \beta_{k}(t) w_{k}(x)
$$

Denote

$$
\begin{aligned}
& \varphi^{(n)}(x)=\sum_{k=1}^{n} \varphi_{k} w_{k}(x), \quad \psi^{(n)}(x)=\sum_{k=1}^{n} \psi_{k} w_{k}(x), \\
& \beta_{k}(0)=\varphi_{k}, \quad \beta_{k}^{\prime}(0)=\psi_{k},
\end{aligned}
$$

the approximate of the functions $\varphi(x)$ and $\psi(x)$. Substituting the approximate solution in equation (1.1), multiplying both sides by $\int_{x}^{1}(h(\xi)-h(x)) w_{i}(\xi) d \xi$, then integrating according to $x$ on $(0,1)$, we get

$$
\begin{aligned}
& \int_{0}^{1}\left(u_{t t}^{(n)}(x, t)-a^{2}(x, t) u_{x x}^{(n)}(x, t)+c(x, t) u^{(n)}(x, t)\right)\left(\int_{x}^{1}(h(\xi)-h(x)) w_{i}(\xi) d \xi\right) d x \\
& =\int_{0}^{1} f(x, t)\left(\int_{x}^{1}(h(\xi)-h(x)) w_{i}(\xi) d \xi\right) d x \\
& \quad+\int_{0}^{1} \int_{0}^{t} \alpha(t-s) K\left(s, u^{(n)}(x, s)\right) d s\left(\int_{x}^{1}(h(\xi)-h(x)) w_{i}(\xi) d \xi\right) d x
\end{aligned}
$$

Substituting (4.1) in (4.3), we get

$$
\begin{aligned}
& \sum_{k=1}^{n} \beta_{k}^{\prime \prime}(t)\left(w_{k}(x), \int_{x}^{1}(h(\xi)-h(x)) w_{i}(\xi) d \xi\right)_{L_{2}(0,1)} \\
& \quad+\sum_{k=1}^{n} \beta_{k}(t)\left[\left(-a^{2}(x, t) w_{k}^{\prime \prime}(x), \int_{x}^{1}(h(\xi)-h(x)) w_{i}(\xi) d \xi\right)_{L_{2}(0,1)}\right. \\
& \left.\quad+\left(c w_{k}(x), \int_{x}^{1}(h(\xi)-h(x)) w_{i}(\xi) d \xi\right)_{L_{2}(0,1)}\right] \\
& =\int_{0}^{1} f(x, t) \int_{x}^{1}(h(\xi)-h(x)) w_{i}(\xi) d \xi d x \\
& \quad+\int_{0}^{1} \int_{0}^{t} \alpha(t-s) K\left(s, \sum_{k=1}^{n} \beta_{k}(s) w_{k}(x)\right) d s \int_{x}^{1}(h(\xi)-h(x)) w_{i}(\xi) d \xi d x
\end{aligned}
$$

Integrating by parts in $L_{2}(0,1)$ the left-hand side of (4.4) yields

$$
\begin{aligned}
\sum_{k=1}^{n} \beta_{k}^{\prime \prime}(t) & \left(h^{\prime}(x) \int_{x}^{1} w_{k}(\xi) d \xi, \int_{x}^{1} w_{i}(\xi) d \xi\right)_{L_{2}(0,1)} \\
& +\sum_{k=1}^{n} \beta_{k}(t)\left(2\left(a a_{x}\right)_{x} w_{k}(x), \int_{x}^{1}(h(\xi)-h(x)) w_{i}(\xi) d \xi\right)_{L_{2}(0,1)} \\
& -\left(\left(4 a a_{x} h^{\prime}+a^{2} h^{(2)}\right) w_{k}(x), \int_{x}^{1} w_{i}(\xi) d \xi\right)_{L_{2}(0,1)}
\end{aligned}
$$




$$
\begin{aligned}
& \quad-a^{2} h^{\prime}\left(w_{k}(x), w_{i}(x)\right)_{L_{2}(0,1)}-\left(c h^{\prime} \int_{x}^{1} w_{k}(\xi) d \xi, \int_{x}^{1} w_{i}(\xi) d \xi\right)_{L_{2}(0,1)} \\
& +\left(c_{x} \int_{x}^{1} w_{k}(\xi) d \xi, \int_{x}^{1}(h(\xi)-h(x)) w_{i}(\xi) d \xi\right)_{L_{2}(0,1)} \\
& =\int_{0}^{1} f(x, t) \int_{x}^{1}(h(\xi)-h(x)) w_{i}(\xi) d \xi d x \\
& \quad+\int_{0}^{1} \int_{0}^{t} \alpha(t-s) K\left(s, \sum_{k=1}^{n} \beta_{k}(s) w_{k}(x)\right) d s \int_{x}^{1}(h(\xi)-h(x)) w_{i}(\xi) d \xi d x .
\end{aligned}
$$

Denote

$$
\begin{aligned}
\theta_{k, i}= & \left(h^{\prime}(x) \int_{x}^{1} w_{k}(\xi) d \xi, \int_{x}^{1} w_{i}(\xi) d \xi\right)_{L_{2}(0,1)} \\
\sigma_{k, i}= & \left(2\left(a a_{x}\right)_{x} w_{k}(x), \int_{x}^{1}(h(\xi)-h(x)) w_{i}(\xi) d \xi\right)_{L_{2}(0,1)} \\
& -\left(\left(4 a a_{x} h^{\prime}+a^{2} h^{(2)}\right) w_{k}(x), \int_{x}^{1} w_{i}(\xi) d \xi\right)_{L_{2}(0,1)} \\
& -a^{2} h^{\prime}\left(w_{k}(x), w_{i}(x)\right)_{L_{2}(0,1)}-\left(c h^{\prime} \int_{x}^{1} w_{k}(\xi) d \xi, \int_{x}^{1} w_{i}(\xi) d \xi\right)_{L_{2}(0,1)} \\
& +\left(c_{x} \int_{x}^{1} w_{k}(\xi) d \xi, \int_{x}^{1}(h(\xi)-h(x)) w_{i}(\xi) d \xi\right)_{L_{2}(0,1)} \\
F_{i}(t)= & \int_{0}^{1} f(x, t)\left(\int_{x}^{1}(h(\xi)-h(x)) w_{i}(\xi) d \xi\right) d x \\
& +\sum_{k=1}^{n} \int_{0}^{1}\left(\int_{0}^{t} \alpha(t-s) K\left(s, \beta_{k}(s) w_{k}(x)\right) d s\right)\left(\int_{x}^{1}(h(\xi)-h(x)) w_{i}(\xi) d \xi\right) d x .
\end{aligned}
$$

then (4.5) becomes

$$
\sum_{k=1}^{n} \beta_{k}^{\prime \prime}(t) \theta_{k, i}+\beta_{k}(t) \sigma_{k, i}=F_{i}(t), \quad \beta_{k}(0)=\varphi_{k}, \quad \beta_{k}^{\prime}(0)=\psi_{k} .
$$

Consequently, we obtain a Cauchy system of second-order integro-differential equations with smooth coefficients, so it has one and only one solution that for every $n$ there exists a unique sequence $u^{(n)}$ that satisfies (4.3).

Lemma 4 The sequence $\left(u^{(n)}\right)$ is bounded.

Proof Multiplying (4.3) by $\beta_{i}^{\prime}(t)$ then summing with respect to $i$ from 1 to $n$ it yields

$$
\begin{gathered}
\int_{0}^{1}\left(u_{t t}^{(n)}(x, t)-a^{2}(x, t) u_{x x}^{(n)}(x, t)+c(x, t) u^{(n)}(x, t)\right)\left(\int_{x}^{1}(h(\xi)-h(x)) u_{t}^{(n)}(\xi, t) d \xi\right) d x \\
=\int_{0}^{1} f(x, t) \int_{x}^{1}(h(\xi)-h(x)) u_{t}^{(n)}(\xi, t) d \xi d x \\
\quad+\int_{0}^{1} \int_{0}^{t} a(t-s) K\left(s, u^{(n)}(x, s)\right) d s\left(\int_{x}^{1}(h(\xi)-h(x)) u_{t}^{(n)}(\xi, t) d \xi\right) d x
\end{gathered}
$$


Guezane-Lakoud et al. Boundary Value Problems 2013, 2013:102

Page 9 of 12

http://www.boundaryvalueproblems.com/content/2013/1/102

Integrating (4.6) over $t$ from 0 to $\tau$ we obtain

$$
\begin{aligned}
& \int_{0}^{1} h^{\prime}(x)\left[c(x, \tau)\left(\int_{x}^{1} u^{(n)}(\xi, \tau) d \xi\right)^{2}+a^{2}(x, \tau)\left(u^{(n)}(x, \tau)\right)^{2}+\left(\int_{x}^{1} u_{t}^{(n)}(\xi, \tau) d \xi\right)^{2} d x\right] \\
& =\int_{0}^{1} h^{\prime}(x)\left[c(x, 0)\left(\int_{x}^{1} \varphi^{(n)}(\xi) d \xi\right)^{2}\right. \\
& \left.\quad+a^{2}(x, 0)\left(\varphi^{(n)}(x)\right)^{2}+\left(\int_{x}^{1} \psi^{(n)}(\xi) d \xi\right)^{2}\right] d x \\
& +\int_{Q^{\tau}}\left[2 h^{\prime} a a_{t}\left(u^{(n)}\right)^{2}+h^{\prime} c_{t}\left(\int_{x}^{1} u^{(n)}(\xi, t) d \xi\right)^{2}\right. \\
& \quad-4\left(a_{x}^{2}+a a_{x x}\right) u^{(n)}\left(\int_{x}^{1}(h(\xi)-h(x)) u_{t}^{(n)}(\xi, t) d \xi\right) \\
& \quad-\left(8 a a_{x} h^{\prime}(x)+2 a^{2} h^{(2)}(x)\right) u^{(n)}\left(\int_{x}^{1} u_{t}^{(n)}(\xi, t) d \xi\right) \\
& +2 c_{x}\left(\int_{x}^{1} u^{(n)}(\xi, t) d \xi\right)\left(\int_{x}^{1}(h(\xi)-h(x)) u_{t}^{(n)}(\xi, t) d \xi\right) \\
& +2 f(x, t)\left(\int_{x}^{1}(h(x)-h(\xi)) u_{t}^{(n)}(\xi, t) d \xi\right) \\
& \left.+2 \int_{0}^{t} \alpha(t-s) K\left(s, u^{(n)}(x, s)\right) d s\left(\int_{x}^{1}(h(x)-h(\xi)) u_{t}^{(n)}(\xi, t) d \xi\right)\right] d x d t .
\end{aligned}
$$

Thanks to Cauchy inequality, $\epsilon$-inequality, the hypotheses on the operator $K$ to the last term in the right-hand side of (4.7), we get

$$
\begin{aligned}
& 2\left|\int_{Q^{\tau}} \int_{0}^{t} a(t-s) K\left(s, u^{(n)}(x, s)\right) d s\left(\int_{x}^{1}(h(\xi)-h(x)) u_{t}^{(n)}(\xi, t) d \xi\right) d x d t\right| \\
& \quad \leq l^{2} \tau\left\|K\left(t, u^{(n)}\right)\right\|_{L^{2}\left(Q^{\tau}\right)}^{2}+4 k_{1}^{2} \int_{Q^{\tau}}\left(\int_{x}^{1} u_{t}(\xi, t) d \xi\right)^{2} d x d t \\
& \quad \leq l^{2} \tau\left\|u^{(n)}\right\|_{L^{2}\left(Q^{\tau}\right)}^{2}+4 k_{1}^{2} \int_{Q^{\tau}}\left(\int_{x}^{1} u_{t}^{(n)}(\xi, t) d \xi\right)^{2} d x d t .
\end{aligned}
$$

Using similar inequalities for the second, the third and the fourth terms in the right-hand side of (4.7), then regrouping the same terms yields

$$
\begin{aligned}
\int_{0}^{1} k_{0} & {\left[\left(\int_{x}^{1} u_{t}^{(n)}(\xi, \tau) d \xi\right)^{2}+a_{0}^{2}\left|u^{(n)}(x, \tau)\right|^{2}+c_{0}\left(\int_{x}^{1} u^{(n)}(\xi, \tau) d \xi\right)^{2}\right] d x } \\
\leq & k_{1}\left[\int _ { 0 } ^ { 1 } \left[C_{0}\left(\int_{x}^{1} \varphi^{(n)}(\xi) d \xi\right)^{2}+A_{0}^{2}\left(\varphi^{(n)}(x)\right)^{2}\right.\right. \\
& \left.\left.+\left(\int_{x}^{1} \psi^{(n)}(\xi) d \xi\right)^{2}\right] d x+2\|f\|_{L_{2}\left(Q^{\tau}\right)}^{2}\right] \\
& +\int_{Q^{\tau}}\left[3 k_{1} C_{1}\left(\int_{x}^{1} u^{(n)}(\xi, t) d \xi\right)^{2}+\left[k_{1}\left(10 A_{0} A_{1}+A_{0}^{2}+A_{1}^{2}\right)+l^{2} \tau\right]\left|u^{(n)}(x, t)\right|^{2}\right. \\
& \left.+\left[k_{1}\left(3 C_{1}+4 A_{0} A_{1}+4 A_{1}^{2}+2\right)+4 k_{1}^{2}\right]\left(\int_{x}^{1} u_{t}^{(n)}(\xi, t) d \xi\right)^{2}\right] d x d t
\end{aligned}
$$


Let $\widetilde{L}=\widetilde{M} / m$, where

$$
\widetilde{M}=k_{1} \max \left(3 C_{1}+4 A_{0} A_{1}+4 A_{1}^{2}+2+4 k_{1}, 10 A_{0} A_{1}+A_{0}^{2}+4 A_{1}^{2}+\frac{l^{2} T}{k_{1}}, C_{0}\right)
$$

then (4.8) becomes

$$
\begin{aligned}
\int_{0}^{1} & {\left[\left(\int_{x}^{1} u^{(n)}(\xi, \tau) d \xi\right)^{2}+u^{(n)}(x, \tau)^{2}+\left(\int_{x}^{1} u_{t}^{(n)}(\xi, \tau) d \xi\right)^{2}\right] d x } \\
\leq & \widetilde{L}\left[\int_{0}^{1}\left[\left(\int_{x}^{1} \varphi^{(n)}(\xi) d \xi\right)^{2}+\left(\varphi^{(n)}(x)\right)^{2}+\left(\int_{x}^{1} \psi^{(n)}(\xi) d \xi\right)^{2}\right] d x+\|f\|^{2}\right. \\
& \left.+\int_{Q^{\tau}}\left[\left(\int_{x}^{1} u^{(n)}(\xi, t) d \xi\right)^{2}+\left|u^{(n)}(x, t)\right|^{2}+\left(\int_{x}^{1} u_{t}^{(n)}(\xi, t) d \xi\right)^{2}\right] d x d t\right]
\end{aligned}
$$

Now, we apply Gronwall lemma to get

$$
\begin{aligned}
& \int_{0}^{1}\left[\left(\int_{x}^{1} u_{t}(\xi, \tau) d \xi\right)^{2}+|u(x, \tau)|^{2}+\left(\int_{x}^{1} u(\xi, \tau) d \xi\right)^{2}\right] d x \\
& \quad \leq e^{\tau \widetilde{L}}\left(\int_{0}^{1}\left[\left(\int_{x}^{1} \varphi^{(n)}(\xi) d \xi\right)^{2}+\left(\varphi^{(n)}(x)\right)^{2}+\left(\int_{x}^{1} \psi^{(n)}(\xi) d \xi\right)^{2}\right] d x+\|f\|^{2}\right)
\end{aligned}
$$

Integrating (4.10) according to $\tau$ on $[0, T]$ yields

$$
\left\|u^{(n)}\right\|_{H(Q)}^{2} \leq T e^{T \widetilde{L}}\left(\left\|\varphi^{(n)}\right\|_{H(0,1)}^{2}+\left\|\psi^{(n)}\right\|^{2}+\|f\|_{L_{2}(Q)}^{2}\right) .
$$

Thus inequality (4.11) implies the boundedness of the sequence $u^{(n)}$.

Remark 5 We have proved that the sequence $\left\{u^{(n)}\right\}$ is bounded, so we can extract a subsequence, which we denote by $\left\{u^{\left(n_{k}\right)}\right\}$ that is weakly convergent. Now we prove that its limit is the desired solution of the problem (1.1)-(1.4).

Lemma 6 The limit of the subsequence $\left\{u^{\left(n_{k}\right)}\right\}$ is the solution of the problem (1.1)-(1.4).

Proof We shall prove that the limit of the subsequence $\left\{u^{\left(n_{k}\right)}\right\}$ satisfies the identity (2.1). Let $\theta_{k}(t) \in C^{2}(0, T)$, such that $\theta_{k}^{\prime}(T)=0$, let us prove that identity (2.1) holds for any functions $v(x, t)=\sum_{k=1}^{n} \theta_{k}(t) w_{k}(x) \in H_{T}(Q)$. Since the set $S_{n}=\left\{v(x, t)=\sum_{k=1}^{n} \theta_{k}(t) w_{k}(x), \theta_{k}(t) \in\right.$ $\left.C^{2}(0, T), \theta_{k}^{\prime}(T)=0\right\}$ is such that $\bigcup_{n=1}^{\infty} S_{n}$ is dense in $H_{T}(Q)$, it suffices to prove (2.1) for $v \in S_{n}$. Multiplying (4.3) by $\theta_{k}^{\prime}(t)$, summing according to $k$ from 1 to $n$, then integrating over $t$ from 0 to $T$, we obtain

$$
\begin{aligned}
\int_{Q}[ & -h^{\prime}(x)\left(\int_{x}^{1} u_{t}^{\left(n_{k}\right)} d \xi\right)\left(\int_{x}^{1} v_{t t}(\xi, t) d \xi\right) \\
& -\left(4 a a_{x} h^{\prime}(x)+a^{2} h^{(2)}(x)\right) u^{\left(n_{k}\right)} \int_{x}^{1} v_{t}(\xi, t) d \xi \\
& -a^{2} h^{\prime}(x) u^{\left(n_{k}\right)} v_{t}-c h^{\prime}(x) \int_{x}^{1} u^{\left(n_{k}\right)}(\xi, t) d \xi \int_{x}^{1} v_{t}(\xi, t) d \xi
\end{aligned}
$$




$$
\begin{aligned}
& +2\left(a a_{x}\right)_{x} u^{\left(n_{k}\right)} \int_{x}^{1}(h(\xi)-h(x)) v_{t}(\xi, t) d \xi \\
& \left.+c_{x}\left(\int_{x}^{1} u^{\left(n_{k}\right)}(\xi, t) d \xi\right)\left(\int_{x}^{1}(h(\xi)-h(x)) v_{t}(\xi, t) d \xi\right)\right] d x d t \\
& =\int_{Q} f(x, t)\left(\int_{x}^{1}(h(\xi)-h(x)) v_{t}(\xi, t) d \xi\right) d x d t \\
& +\int_{Q} \int_{0}^{t} \alpha(t-s) K\left(s, u^{\left(n_{k}\right)}(x, s)\right) d s\left(\int_{x}^{1}(h(\xi)-h(x)) v_{t}(\xi, t) d \xi\right) d x d t .
\end{aligned}
$$

Denote by $u$ the weak limit of the subsequence $\left\{u^{\left(n_{k}\right)}\right\}$ when $k$ tends to infinity. Hence,

$$
\begin{aligned}
& \mid \int_{Q} \int_{0}^{t} \alpha(t-s) K\left(s, u^{\left(n_{k}\right)}(x, s)\right) d s\left(\int_{x}^{1}(h(\xi)-h(x)) v_{t}(\xi, t) d \xi\right) d x d t \\
& \quad-\int_{Q} \int_{0}^{t} \alpha(t-s) K(s, u(x, s)) d s\left(\int_{x}^{1}(h(\xi)-h(x)) v_{t}(\xi, t) d \xi\right) d x d t \mid \\
& =\mid \int_{Q}\left(\int_{0}^{t} \alpha(t-s)\left(K\left(s, u^{\left(n_{k}\right)}(x, s)\right)-K(s, u(x, s))\right) d s\right) \\
& \quad \times\left(\int_{x}^{1}(h(\xi)-h(x)) v_{t}(\xi, t) d \xi\right) d x d t \mid \\
& \leq 2 k l \sqrt{T}\left(\int_{Q}\left(\int_{x}^{1}\left|v_{t}\right| d \xi\right)^{2} d x d t\right)^{1 / 2} \\
& \quad \times\left(\int_{Q}\left|K\left(s, u^{\left(n_{k}\right)}(x, s)\right)-K(s, u(x, s))\right|^{2} d s d x\right)^{1 / 2} \\
& =2 k l \sqrt{T}\left(\int_{Q}\left(\int_{x}^{1}\left|v_{t}\right| d \xi\right)^{2} d x d t\right)^{1 / 2}\left\|K\left(t, u^{\left(n_{k}\right)}\right)-K(t, u)\right\|_{L^{2}(Q)} \\
& \leq 2 k l \sqrt{T}\left(\int_{Q}\left(\int_{x}^{1}\left|v_{t}\right| d \xi\right)^{2} d x d t\right)^{1 / 2}\left\|u^{\left(n_{k}\right)}-u\right\|_{L^{2}(Q)} \longrightarrow 0 .
\end{aligned}
$$

Finally, by passing to the limit in (4.12), we get that the limit $u$ satisfies (2.1).

Example 7 Consider the following boundary value problem for hyperbolic integrodifferential equation for $0<x<1,0<t \leq T$ :

$$
\begin{aligned}
\frac{\partial^{2} u}{\partial t^{2}}(x, t)-\frac{1}{8} \frac{\partial^{2} u}{\partial x^{2}}(x, t)+\frac{1}{2} \pi^{2} u(x, t) \\
=2 \pi^{2} \cos (2 \pi x) e^{-\pi t}-\left(t+\frac{1}{\pi}\left[e^{-\pi t}-1\right]\right) \frac{\sin (2 \pi x)}{2 \pi^{2}} \\
\quad+\int_{0}^{t}(t-s)\left(\int_{0}^{x} u(r, s) d r\right) d s,
\end{aligned}
$$

subject to the initial conditions

$$
u(x, 0)=\cos (2 \pi x), \quad u_{t}(x, 0)=-\pi \cos (2 \pi x), \quad 0<x<1
$$


and the weighted integral condition

$$
\begin{aligned}
& \int_{0}^{1} u(x, t) d x=0, \\
& \int_{0}^{1} h(x) u_{t}(x, t) d x=0,
\end{aligned}
$$

where $h(x)=1-\cos (\pi x)$. It is easy to prove that assumptions (H1)-(H3) are satisfied, then from Theorems 2 and 3, and we deduce that the problem (4.13)-(4.16) has a unique generalized solution in the sense of Definition 1. Moreover, the function $u(x, t)=e^{-\pi t} \cos (2 \pi x)$ is the solution of this problem.

\section{Competing interests}

The authors declare that they have no competing interests.

\section{Authors' contributions}

All authors typed, read and approved the final manuscript.

\section{Author details}

${ }^{1}$ Faculty of Sciences, Laboratory of Advanced Materials, Badji Mokhtar-Annaba University, P.O. Box 12, Annaba, 23000, Algeria. ${ }^{2}$ Faculty of Sciences, Laboratory LASEA, Badji Mokhtar-Annaba University, P.O. Box 12, Annaba, 23000, Algeria.

\section{Acknowledgements}

The authors would like to thank the referees for their valuable suggestions.

Received: 23 November 2012 Accepted: 10 April 2013 Published: 24 April 2013

\section{References}

1. Beilin, SA: Existence of solutions for one dimensional wave equations with nonlocal conditions. Electron. J. Differ. Equ. 76, 1-8 (2001)

2. Cannon, JR: The solution of the heat equation subject to the specification of energy. Q. Appl. Math. 21, 155-160 (1963)

3. Dehghan, M: On the solution of an initial-boundary value problem that combines Neumann and integral condition for the wave equation. Numer. Methods Partial Differ. Equ. 21, 24-40 (2005)

4. Dehghan, M: A finite difference method for a non-local boundary value problem for two dimensional heat equation. Appl. Math. Comput. 112, 133-142 (2000)

5. Ionkin, NI: Solutions of boundary value problem in heat conduction theory with nonlocal boundary conditions. Differ. Uravn. (Minsk) 13, 294-304 (1977)

6. Dubey, RS: Existence of the unique solution to abstract second order semilinear integrodifferential equations. Nonlinear Dyn. Syst. Theory 10(4), 375-386 (2010)

7. Crandall, MG, Souganidis, P: Convergence of difference approximations of quasilinear evolution equations. Nonlinear Anal. 10, 425-445 (1986)

8. Cannon, JR, Lin, Y, Wang, S: An implicit finite difference scheme for the diffusion equation subject to mass specification. Int. J. Eng. Sci. 28, 573-578 (1990)

9. Dehghan, M: Second order schemes for a boundary value problem with Neumann boundary conditions. Appl. Math. Comput. 138, 173-184 (2002)

10. Bahuguna, D: Quasilinear integrodifferential equations in Banach spaces. Nonlinear Anal. 24, 175-183 (1995)

11. Kumar, K, Kumar, R, Shukla, RK: Nonlocal parabolic integro-differential equations with delay. Int. J. Appl. Math. Res. 1(4), 549-564 (2012)

12. Guezane-Lakoud, A, Chaoui, A: Rothe method applied to semilinear hyperbolic integro-differential equation with integral conditions. Int. J. Open Probl. Comput. Sci. Math. 4, 1-14 (2011)

13. Dabas, J, Bahuguna, D: An integro-differential equation with an integral boundary condition. Math. Comput. Model. 50, 123-131 (2009)

14. Guezane-Lakoud, A, Dabas, J, Bahuguna, D: Existence and uniqueness of generalized solutions to a telegraph equation with an integral boundary condition via Galerkin method. Int. J. Math. Math. Sc 2011, Article ID 451492 (2011)

15. Guezane-Lakoud, A, Bendjazia, N: Galerkin method for solving a telegraph equation with a weighted integral condition. Int. J. Open Probl. Complex Anal. 5, 41-53 (2012)

doi:10.1186/1687-2770-2013-102

Cite this article as: Guezane-Lakoud et al.: Galerkin method applied to telegraph integro-differential equation with a weighted integral condition. Boundary Value Problems 2013 2013:102. 